\title{
Le comité d'experts en médecine des voyages
}

\section{Christoph Hatz}

Président de la Société suisse de Médecine Tropicale et des Médecine Voyages, Institut Tropical Suisse, Bâle, et Institut de médecine sociale et préventive de l'université de Zurich
Correspondance:

Prof. Christoph Hatz STI

Socinstrasse 57

Case postale

CH-4002 Bâle

Tél. 0612848255

christoph.hatz@unibas.ch
Derrière ce nom on trouve un concept destiné à améliorer le conseil en médecine des voyages. Initié il y a près de 30 ans, ce concept est mis en pratique et développé sans relâche. Les centres de médecine des voyages de Zurich, Bâle, Genève, Lausanne, Berne et St-Gall ont élaboré des recommandations communes pour une information uniforme et basée sur des données scientifiques à l'intention des personnes qui se rendent dans des pays tropicaux et subtropicaux. En leur qualité de membres, la compagnie aérienne Swiss et la société de médecine générale veillent à transmettre une information claire et uniforme aux voyageurs suisses. Les membres du comité travaillent bénévolement, ce qui leur permet d'éviter tout conflit d'intérêt. Les recommandations sont publiées par l'Office fédéral de la santé publique et mises à jour chaque semaine sur l'Internet. Vous les obtenez en cliquant sur www.safetravel.ch et www.tropimed.com.

$\mathrm{Au}$ fil des ans, les activités du comité ont permis d'améliorer grandement le conseil donné en Suisse en matière de médecine des voyages. Dans une étude comparative, on a montré que les médecins suisses prodiguaient des conseils nettement mieux étayés que leurs collègues allemands qui ne disposaient pas d'informations semblables. C'est l'une des raisons pour lesquelles des leaders d'opinion d'Allemagne, d'Autriche, de France, des Pays-Bas, de Grande-Bretagne et de Suède ont rejoint le comité d'experts. On a ainsi élaboré une recommandation pratiquement identique en Suisse, en Autriche et en Allemagne pour se protéger contre la malaria.

Bien que le comité d'experts pour la médecine des voyages n'ait aucun statut officiel, ses membres ont contribué ces trente dernières années à ce que les personnes germanophones puissent, pour le conseil en médecine des voyages, s'appuyer sur un avis consensuel basé sur des données scientifiques et émis par des experts, et donc mieux évaluer les risques pour leur santé et s'en protéger.

\section{La Société Suisse de Médecine Tropicale et Médecine des Voyages}

\section{Stephan Koch}

Vice-président de la Société Suisse de Médecine Tropicale et Médecine des Voyages

\section{Correspondance: Dr Stephan Koch Zentralstrasse $55 a$ CH-5610 Wohlen Tél. 0566187007}

\section{st.koch@spiderweb.ch}

www.tropenmedizin-fmh.ch
Fondée il y a trente ans, la Société Suisse de Médecine tropicale a fortement modifié ses objectifs depuis lors. La médecine tropicale porte sur l'épidémiologie, la santé publique, la prévention, le diagnostic ainsi que le traitement des maladies tropicales et maladies des voyages. Un meilleur diagnostic permet de détecter plus vite les maladies infectieuses, de les traiter et d'éviter qu'elles ne se répandent. Comme la mobilité de la population au cours des dernières décennies a fortement augmenté, la société s'occupe aujourd'hui surtout de mesures préventives et cliniques en médecine des voyages. Des médecins au bénéfice d'une expérience de plusieurs années à l'étranger offrent leurs conseils aux médecins de famille en cabinet privé et aux médecins hospitaliers pour le diagnostic différencié de maladies consécutives à un voyage. Grâce à la collaboration des spécialistes en médecine tropicale, le comité d'experts en médecine des voyages a pu unifier le conseil dans le domaine de la vaccination en Suisse et dans d'autres pays européens.
La Société Suisse de Médecine Tropicale et Médecine des Voyages encourage la formation postgraduée des jeunes médecins et la formation continue de ses membres; elle est partie de la Société Suisse de Médecine Tropicale et Parasitologie.

La recherche dans le domaine de la médecine tropicale porte entre autres sur des thèmes relevant de la santé publique telle que la médecine des migrations (neglected diseases) et (diseases among neglected people) dans les pays en voie de développement en vue de l'accès aux soins de santé, souvent impossible ou difficile pour cette population. Par conséquent, de nombreuses maladies infectieuses ou de médecine générale ne sont souvent pas détectées ou trop tard chez les migrants et les sans-papiers. Notre société cherche à remédier à ces situations en développant des réseaux en Europe et dans le monde entier. Des mesures visant la prise de conscience au niveau politique permettent en outre d'élaborer des projets porteurs d'avenir. 